\title{
Microfluidics: the great divide
}

\author{
Nathan Blow
}

\begin{abstract}
Although many intricate microfluidic devices have been created in academic laboratories around the world, far fewer have been commercialized for wider use. But several efforts are underway to bridge this divide.
\end{abstract}

\begin{abstract}
Abraham Lee noticed something curious about the field of microfluidics when he was working for the Defense Advanced Research Project Agency (DARPA). When he looked around, he could see that a number of brilliant researchers and engineers were coming up with innovative microfluidic devices, fabrication strategies and technologies in their labs. But he also noticed that many of these same researchers tended not to collaborate with one another.
\end{abstract}

Lee thought the best approach to advancing the field and designing better microfluidic devices would be through the creation of a collaborative center focused on microfluidic-based technology development. So when he left DARPA to join the faculty of the University of California, Irvine, he also helped start the Micro/nano Fluidics Fundamentals Focus (MF3) Center, which is now home to several academic groups who share ideas, expertise and tools.

In many respects the MF3 Center is not unique; in recent years there have been a growing number of nanotechnology/ microfluidics centers springing up around the world. What does make Lee's center different, however, is that they are also partnering with several commercial developers in an attempt to speed the transition from the design stage to commercialization. "Academics are doing the fundamental development," says Lee, "but they tend to be random and not focused. The commercial people can tell you that these are the key bottlenecks and hurdles we are facing when it comes to commercialization."

According to Holger Becker, who is the

\section{cofounder and chief scientific officer at} the microfluidics company Microfluidic ChipShop $\mathrm{GmbH}$, such collaborative efforts and interactions are occurring at the perfect moment in time. "An intense discussion has now started concerning the widening gap between what is developing in academia and the needs and the actions in industry," he says.

Although many developers and companies have spent many years searching for a microfluidics 'killer app' that would lead to the wider adoption of microfluidics technologies, several developers are now moving away from the idea of the single 'killer app' and are instead focusing their efforts on bridging the divide between industry and academia in the hopes of using microfluidics as an enabling technology for a wide range of life science applications.

\section{Creating a bridge}

One of the major goals of the MF3 Center is to develop microfluidic modules for various tasks_-for example, cell sorting or fluid transport— - so that a variety of well-designed modules are available to the various researchers working at the center.

"In the past I would go in with all the tools I knew, but I am not good at everything [when it comes to microfluidic fabrication and design]. So in the end, I would put together a device that was very good in one aspect, but might have been mediocre in another," says Lee. By having expertise in different areas of microfabrication and chip design, Lee and others at the center hope to create more robust microfluidic devices at a faster pace.

The other component of the center's mission is to design platforms and modules that can be quickly converted to large-scale manufacturing. Lee says that one of the companies associated with the center, Douglas Machining, has expertise in the mass production approach of reel-to-reel printing, so several efforts are underway now to use fabrication approaches, such as lithography

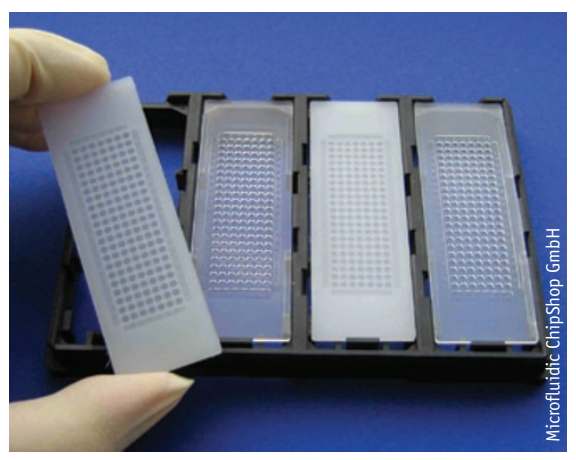

Microtiter plates can often be used to contain several microfluidic devices. 


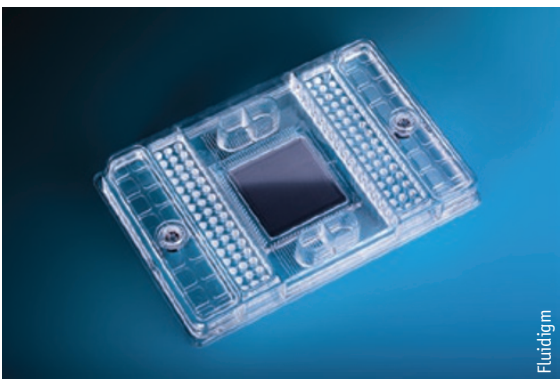

The Fluidigm Access Array IFC, used for sample preparation for next-generation sequencing, is an example of the emergence of preparative microfluidic devices.

or embossing, that are amenable to reelto-reel printing in the development of new microfluidic platforms. Most projects are in fact developed and designed from the outset to be compatible with the center's manufacturing competence. "In this way, if we have a technology, there is a straight path to manufacturing," explains Lee.

Several companies are establishing closer ties with academic labs as well in an effort to speed development. Nano Terra is a new company working directly with other companies to solve microfluidic and nanotechnology problems. Founded in 2005 by Myer Berlow, a former executive at the internet service provider AOL, and George Whitesides, a professor at Harvard University, along with entrepreneur Carmichael Roberts and technology executive Ueli Morant, Nano Terra works with larger companies in a collaborative model to develop microfluidic technologies as well as identify areas of opportunity in which nanotechnology and microfluidic approaches could make a difference for researchers and clinicians. According to Berlow, when he first looked at the field, he had very similar thoughts to Lee, noting the need for more cooperation among scientists to spread the use of microfluidic approaches. "Academics or technology innovators need to listen to and cooperate with the big manufacturers and vice versa."

Although microfluidic technology is slowly making inroads into basic research labs, Berlow suspects that its wider adoption, especially in the medical field, might initially occur in unexpected places. "I think it will be much easier to see microfluidicsbased medical devices in the third world because there is great need," he says. Lee agrees, but still sees challenges in getting robust, inexpensive microfluidic devices into the field. Both Berlow and Lee do note that a major step in the development of truly inexpensive microfluidic devices was accomplished in Whitesides' lab last year when his group used layers of paper and tape to construct a three-dimensional microfluidic device ${ }^{1}$. Their prototype device cost $\$ 0.03$ to build and was capable of testing up to four different samples for four different analytes and displaying the results of the assay.

\section{Lost and found in translation}

"If you go back ten years, there has been a lot of promise when it comes to microfluidics but not a lot of delivery," says Gajus Worthington, chief executive officer at Fluidigm, a company that has developed microfluidic-based applications using multilayer soft lithography fabrication approaches pioneered by Stephen Quake's group at Stanford University.

Analytical applications such as genotyping, gene expression analysis and digital PCR have been enabled and enhanced through the use of soft lithography-fabricated microfluidic platforms that allow the creation of many channels and valves on a chip. But Worthington says moving beyond these initial applications has proved to be a challenge: "Going from creating very good analytical tools, which I think are well established now, to devices that are truly preparative, was a harder problem.” This problem is starting to be solved, according to many developers.

Marc Unger, chief scientific officer at Fluidigm, says one such complicated application for which scientists are increasingly interested in developing chips is examining single cells. "Researchers came to the basic realization that looking at the average of a group of cells is not adequate if you want to understand what is governing their behavior," he says. This has led a growing number of researchers and developers to design microfluidic chips for single-cell applications, moving away from the analytical studies to preparative applications in which cells and macromolecules can be separated on the chip and then recovered after processing.

The idea of preparative microfluidic devices is not new: several such devices have been developed in academic labs over the past few years. In fact, Quake's lab created a chip that could perform automated cell culture, from loading stem cells onto the chip to treating them with different growth factors and growing the cells long enough to assess their differentiation ${ }^{2}$."That work turned into a paper, but it does not enable other folks in the stem cell community to do the same thing," says Unger, highlighting the translation issue for scientists when it comes to complicated microfluidic devices. His research group at Fluidigm is currently working to expand on Quake's efforts by developing a versatile, manufacturable microfluidic chip and instrumentation that will be able to culture individual stem cells in parallel under a range of conditions and media, an effort supported by a grant from the California Institute of Regenerative Medicine.

Lee's own research group at the University of California, Irvine is also working on developing a microfluidic chip for continuous cell-sorting applications. His group has relied on the technique of dielectrophoresis, or the dielectric character of the cell's surface, to sort cell populations on a chip $^{3}$. The challenge for Lee's group when it came to performing continuous cell sorting with a microfluidic device was in the

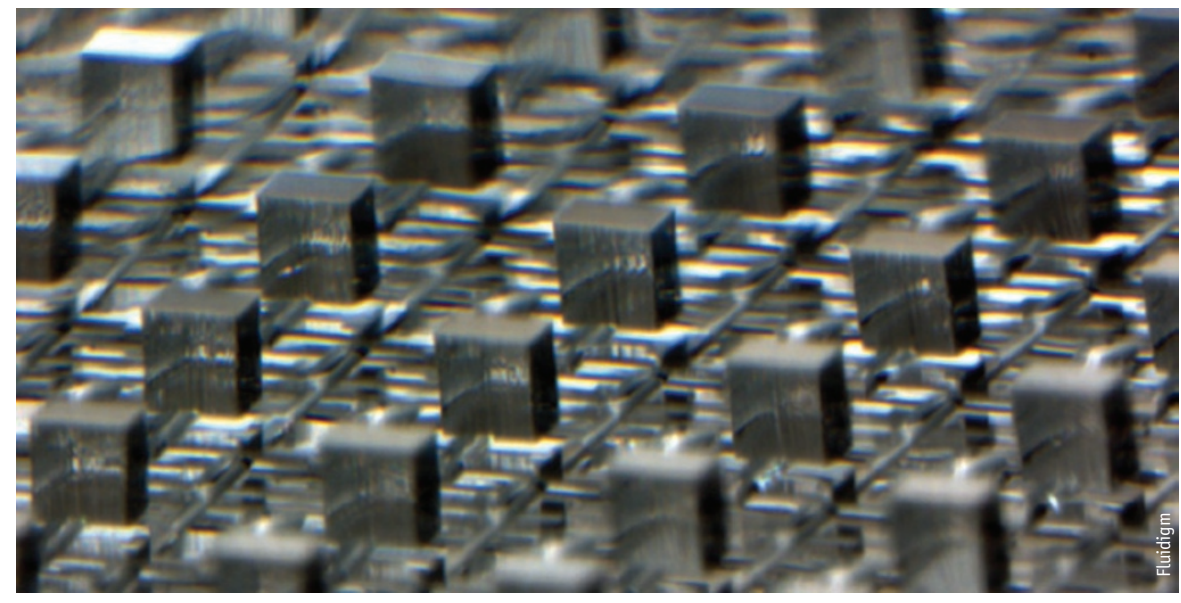

Image of a microfluidic chip taken with a microscope. 


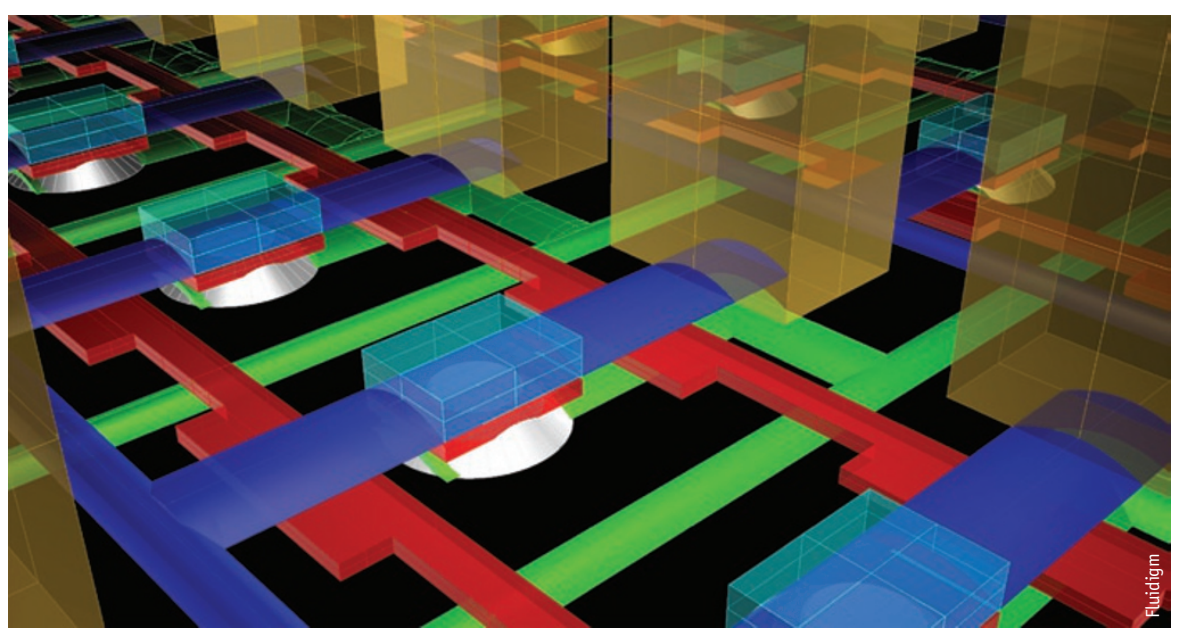

Fluidigm relies on the process of multilayer soft lithography to generate channels and valves when designing microfluidic devices.

microfabrication of a vertical electrode to generate the right and left forces they needed. "Microfabrication is always finicky and takes time," notes Lee, whose group worked to determine the dielectric properties of various cell types while solving the issues of vertical electrode fabrication.

Lee and his medical school collaborators are now working with a company to develop his continuous sorting chip design for possible commercialization. "We are making a strong effort to help them figure out the best way to simplify the process," explains Lee. A big part of that effort is helping to identify the minimal functionality required to make this design useful to researchers and not too complicated to manufacture in large scale.

Although new microfluidic chips and platforms for cell culture and manipulation are on the horizon, Worthington suspects that an increasing number of preparative chips for genetic applications will appear even sooner. Fluidigm has launched a microfluidic chip for sequence enrichment using a PCR approach by modifying its targeted 48.48 array with the addition of an output channel to collect sample before next-generation sequencing. Other companies, including RainDance Technologies, are using similar approaches for sequence enrichment, while several companies, including Agilent Technologies, are now using microfluidics for the upstream sample processing steps before mass spectrometry analysis.

The question of when, or if, the so-called 'killer app' will come along and move microfluidics forward remains on the minds of many technology developers at the moment. "Any new field likes to find a "killer app' because it gives attention to the field," notes Holger Becker. But that has not happened with microfluidics as yet. Although Becker suspects that such an application still might come along one day, in the meantime many developers are starting to view microfluidics as an important enabling technology for a growing number of products and applications_a potentially 'killer idea'.

1. Martinez, A.W., Philips, S.T. \& Whitesides, G.M. Three-dimensional microfluidic devices fabricated in layered paper and tape. Proc. Natl. Acad. Sci. USA 105, 19606-19611 (2008).

2. Gomez-Sjoberg, R., Leyrat, A.A., Pirone, D.M., Chen, C.S. \& Quake, S.R. Versatile, fully automated, microfluidic cell culture system. Anal. Chem. 79, 8557-8563 (2007).

3. Flanagan, L.A. et al. Unique dielectric properties distinguish stem cells and their differentiated progeny. Stem Cells 26, 656-665 (2008).

Nathan Blow is the technology editor for Nature and Nature Methods

(n.blow@boston.nature.com). 
SUPPLIERS GUIDE: COMPANIES OFFERING MICROFLUIDIC

SOLUTIONS

\section{Company}

Abbott Laboratories

Advanced Liquid Logic

Agilent Technologies

Applied Biosystems

ArrayJet

Biacore

Bio-Rad

BioTrove

Caliper Life Sciences

Cellix

Cepheid

Dionex

Dolomite

Eksigent Technologies

febit

Fluidigm

Fluigent

Fluxion Biosciences

Gyros

HandyLab Inc.

Helicos Bioscience Corporation

Hewlett-Packard

Invitrogen

LabSmith

Microchip Biotechnologies Inc

Microdrop Technologies GmbH

Microfluidic ChipShop GmbH

Microfluidics Corp.

Micronics

Micronit Microfluidics

Monogram Biosciences

Nano Terra

Nanobiosym

Nanogen

NetBio

Pyrosequencing $A B$

RainDance Technologies

Roche 454

SpinX Technologies

Surface Logix

Tecan

thinXXS

Wasatch Microfluidics
Web address

http://www.abbott.com/

http://www.liquid-logic.com/

http://www.agilent.com/

http://www.appliedbiosystems.com/

http://www.arrayjet.co.uk/

http://www.biacore.com/

http://www.bio-rad.com/

http://www.biotrove.com/

http://www.caliperlifesciences.com/

http://www.cellixltd.com/

http://www.cepheid.com/

http://www.dionex.com/

http://www.dolomite-microfluidics.com/

http://www.eksigent.com/

http://www.febit.com/

http://www.fluidigm.com/

http://www.fluigent.com/

http://www.fluxionbio.com/

http://www.gyros.com/

http://www.handylab.com/

http://www.helicosbio.com/

http://www.hp.com/

http://www.invitrogen.com/

http://www.labsmith.com/

http://www.microchipbiotech.com/

http://www.microdrop.de/

http://www.microfluidic-chipshop.com/

http://www.microfluidicscorp.com/

http://www.micronics.net/

http://www.micronit.com/

http://www.monogrambio.com/

http://www.nanoterra.com/

http://www.nanobiosym.com/

http://www.nanogen.com/

http://www.networkbiosystems.com/

http://www.pyrosequencing.com/

http://www.raindancetechnologies.com/

http://www.roche.com/

http://www.spinx-technologies.com/

http://www.surfacelogix.com/

http://www.tecan.com/

http://www.thinxxs.com/

http://www.microfl.com/ 